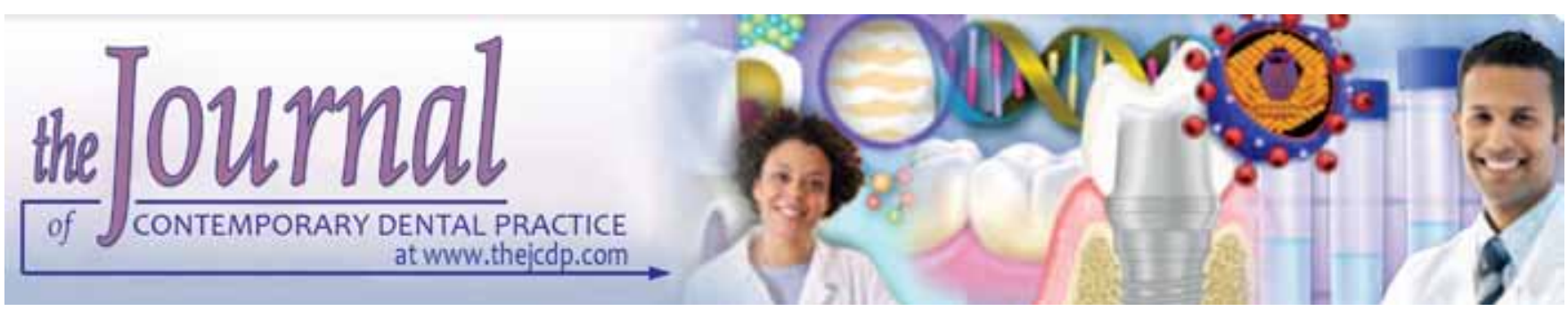

\title{
Repeatability in Color Measurements of a Spectrophotometer using Different Positioning Devices
}

\author{
${ }^{1}$ Michael Hemming, ${ }^{2}$ So Ran Kwon, ${ }^{3}$ Fang Qian
}

\begin{abstract}
Aim: This study aimed to evaluate the repeatability of color measurements of an intraoral spectrophotometer with the use of three different methods by two operators.
\end{abstract}

Materials and methods: A total of 60 teeth were obtained, comprising 30 human maxillary teeth [central incisors $(n=10)$; canines $(n=10)$; molars $(n=10)$ ] and 30 artificial teeth [lateral incisors $(n=10)$; premolar $(n=20)$ ]. Multiple repeated color measurements were obtained from each tooth using three measuring methods by each of the two operators. Five typodonts with alternating artificial and human teeth were made. Measurements were taken by two operators with the Vita EasyShade spectrophotometer using the custom tray (CT), custom jig (CJ) and free hand (FH) method, twice, at an interval of 2 to 7 days. Friedman test was used to detect difference among the three color measuring methods. Post hoc Wilcoxon signed-rank test with Bonferroni correction applied was used for pair-wise comparison of color measurements among the three methods. Additionally, a paired-sample t-test was used to assess a significant difference between the two duplicated measurements made on the same tooth by the same operator for each color parameter and measuring method.

Results: For operator $\mathrm{A}$, mean (SD) overall color change- $\Delta \mathrm{E}^{*}$ (SD) perceived for FH, CT and CJ were 2.21(2.00), 2.39 (1.58) and 2.86 (1.92), respectively. There was statistically significant difference in perceived $\Delta \mathrm{E}^{*}$ in $\mathrm{FH}$ vs $\mathrm{CJ}(\mathrm{p}=0.0107)$. However, there were no significant differences between $\mathrm{FH}$ and CT $(p=0.2829)$ or between CT and CJ $(p=0.1159)$. For operator

\footnotetext{
${ }^{1}$ Department of Dentistry, University of lowa, College of Dentistry, lowa City, USA

${ }^{2}$ Department of Center for Dental Research, Loma Linda University, School of Dentistry, Loma Linda, California, USA

${ }^{3}$ Department of Preventive and Community Dentistry and Division of Biostatistics and Research Design, University of lowa, College of Dentistry, lowa City, USA

Corresponding Author: So Ran Kwon, Associate Professor Center for Dental Research, Loma Linda University, School of Dentistry, 11175 Campus Street Suite A 1010, Loma Linda-92350 California, USA, Phone: 909558 8069, e-mail: sorankwon@ llu.edu
}

B mean $\Delta E^{*}(S D)$ for $F H, C T$ and $C J$ were 3.24 (3.46), 1.95 (1.19) and 2.45 (1.56), respectively. There was a significant difference between FH and CT $(p=0.0031)$. However, there were no statistically significant differences in $\Delta \mathrm{E}^{*}$ in $\mathrm{FH}$ vs CJ ( $p=0.3696)$ or CT vs CJ $(p=0.0809)$.

Conclusion: The repeatability of color measurements was different among the three measuring methods by operators. Overall, the CT method worked well for both operators.

Clinical significance: The use of a custom tray with apertures can improve the repeatability of color measurements of an intraoral spectrophotometer.

Keywords: Custom jig, Custom tray with apertures, Tooth color measurements, Tooth whitening.

How to cite this article: Hemming M, Kwon SR, Qian F. Repeatability in Color Measurements of a Spectrophotometer using Different Positioning Devices. J Contemp Dent Pract 2015;16(12):933-938.

Source of support: Nil

Conflict of interest: None

\section{INTRODUCTION}

It is pivotal to determine baseline color and changes in tooth color to achieve satisfactory esthetic results in restorative dentistry in a reliable and efficient manner. However, the concept of color is difficult to understand and many times not easy to define, and often related more to art than science. ${ }^{1}$ Tooth color measurements are performed clinically with an appropriate shade guide to match existing teeth. Despite the widespread use of shade guides in the clinical practice, it is important to point out that this visual evaluation is subjective and influenced by observer, type of light source and the surrounding environment. Recent advances in technology have enabled the use of specialized devices for shade determination. Clinical studies have shown that technology-based shade determination is more accurate and more consistent than human shade assessment. ${ }^{2,3}$ However, there is still a great 
need to improve the repeatability of these devices in the oral environment. ${ }^{4}$

In color science, evaluation of measurement uncertainties of shade-matching instruments is performed through precision and accuracy testing. Precision is tested by evaluation of repeatability (same method, operator or instrument) and reproducibility (different method, operator and/or instrument). ${ }^{5}$ In a study that compared five dental color-measuring devices, the authors found that the EasyShade and Ikam system were the most reliable in a clinical setting while the other tested devices were more reliable in vitro than in vivo. ${ }^{3}$ In another in vitro study, a varying degree of reliability and accuracy was reported for a color measuring system depending on the type of shade guide system used. ${ }^{6}$ When the precision of measurement of different tooth areas was evaluated, the middle third of each labial tooth surface exhibited the most consistent results. ${ }^{7}$ Since, spectrophotometers can detect small differences in color at a level that is not appreciable by the human eye, an important issue of color science in dentistry is to establish a method to improve operator repeatability of these intraoral devices.

Generally, based on manufacturer's direction of intraoral spectrophotometers the color-measuring probe is placed perpendicular to the facial surface at the middle third of the tooth. Henceforth, subtle changes in direction and placement may contribute to inaccuracy of the measurements. In order to improve the repeatability of measurements the use of a custom fabricated jig made of clear silicone registration material had been proposed. ${ }^{8}$ The authors found that this device provided not only the repeatability of measuring area but also a fixed perpendicular positioning of the instrument. The jig enabled a short-term repeatability of $\Delta \mathrm{E}_{\mathrm{ab}}^{*}<0.7$, which is regarded as not appreciable by the human eye. However, in the study the jig was used for measurement of a single tooth and the feasibility of using this jig on multiple teeth has not been evaluated. An alternative device for controlling the measuring area is a thin, vacuum formed stent with an open aperture that can be easily fabricated for multiple tooth color measurements. It is predicted to produce equal results in terms of repeatability as well as cut back on working time for the clinician making it a more convenient procedure while maintaining the integrity of the measurement.

Different studies have been performed to compare the repeatability of different intraoral spectrophotometers. The aim of this study was to evaluate the repeatability of an intraoral spectrophotometer with the use of two different types of positioning devices; custom fabricated jigs (CJ) and custom trays (CT) compared to the conventional hand held method (FH) on dental typodonts mounted with a combination of extracted human teeth and artificial typodont teeth. The hypotheses to be tested were that first, there will be no difference in the duplicate color parameter $\left(\mathrm{L}^{*}, \mathrm{a}^{*}, \mathrm{~b}^{*}\right)$ measurements regardless of the measuring method used by each operator; second, there will be no difference in color measurements using different measuring methods by each operator; third, there will be no difference in color measurements using different measuring methods by type of tooth material (human teeth $v$ s typodont teeth) and by each operator.

\section{MATERIALS AND METHODS}

Sample selection and typodont mounting: A total of 60 teeth were obtained, comprising 30 human maxillary teeth (central incisors $\mathrm{n}=10$; canines $\mathrm{n}=10$; molars $\mathrm{n}=10$ ) and 30 artificial teeth (lateral incisors $\mathrm{n}=10$; premolar $\mathrm{n}=20$ ). The overall study design is illustrated in Flow Chart 1. Five typodonts (Kavo Dental GmbH, Biberach/ Riß, Germany) with alternating artificial and human teeth were made as shown in Figure 1.

Tooth color measurement: Multiple repeated color measurements were obtained from each tooth using three measuring methods by each of the two dental student operators (A and B). Each operator performed tooth color measurements with an intraoral contact type spectrophotometer (Vita EasyShade, VITA Zahnfabrik, Bad Sackingen, Germany) using the CJ, CT, and FH method twice, at an interval of 2 to 7 days. Custom jigs were fabricated for each typodont to provide repositioning and measurement on the middle third of the facial tooth surface. The jigs were fabricated by attaching a $5 \mathrm{~mm}$ diameter acrylic rod to the middle third of the tooth using lightcured flowable resin (Estelite Flow Quick, Tokuyama Corporation, Tokyo, Japan). Clear silicone registration

Flow Chart 1: Study design

Maxillary typodonts $(n=5)$ with mounted teeth $(n=60)$ (combination of six natural and six artificial teeth by typodont)

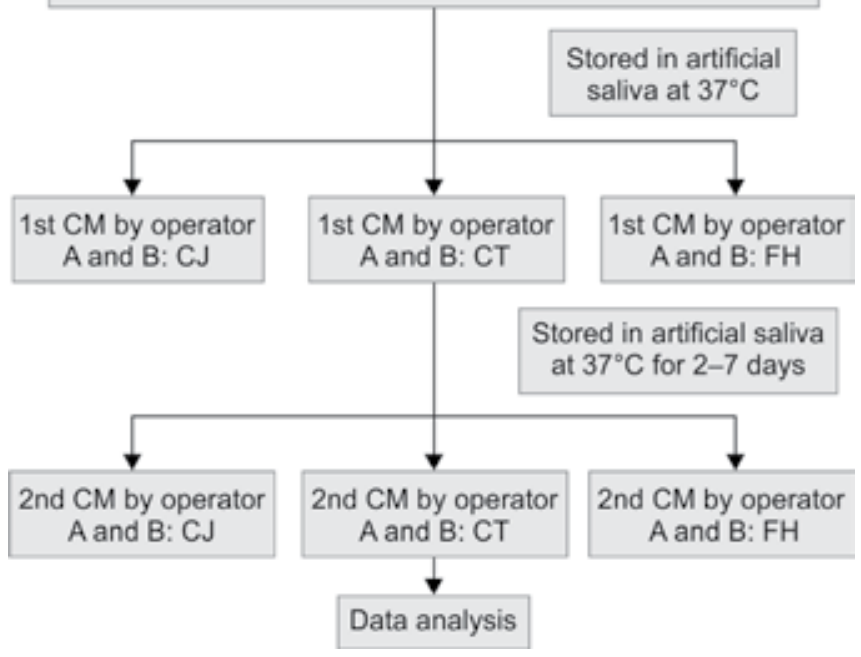


material (ClearBite, Discus Dental, Kulver City, LA, USA) was injected around the cylinder and along the incisal surface of four teeth. Upon setting of the registration material, the rods were simultaneously dislodged from the tooth while the jig was removed from the typodont. ${ }^{8}$ Tooth color measurement with the use of the custom fabricated jig is shown in Figure 2. Custom trays were made with soft vinyl sheets of $1 \mathrm{~mm}$ thickness (Biostar, Great Lakes Orthodontics Ltd., Tonawanda, NY, USA) that was heated on a heat pressure machine (Ministar, Great Lakes Orthodontics Ltd., Tonawanda, NY, USA) and formed to fabricate a custom tray. The tray was separated from the model and trimmed along the gingival margins and trimmed at the middle third of the facial tooth surface to produce an aperture of $6.0 \mathrm{~mm}$ diameter (Fig. 3). The free hand held method was following manufacturer's directions by placing the probe perpendicular to the facial surface at the middle third of the tooth (Fig. 4).

Data Analysis: Paired-sample t-test was performed to detect the difference between the first and second measurements of the three color parameters: $\mathrm{L}^{*}, \mathrm{a}^{*}$, and $b^{*}$ by operator for evaluating the repeatability of measurements. Due to the lack of normality, Friedman test was used to detect difference among the three color measuring methods by evaluating the overall color difference of the first and second measurement as calculated by the following equation: $\Delta \mathrm{E}^{*}=\left[\left(\mathrm{L}_{2}-\mathrm{L}_{1}\right)^{2}+\right.$ $\left.\left(a_{2}-a_{1}\right)^{2}+\left(b_{2}-b_{1}\right)^{2}\right]^{1 / 2}$. Post-hoc Wilcoxon signed-rank test

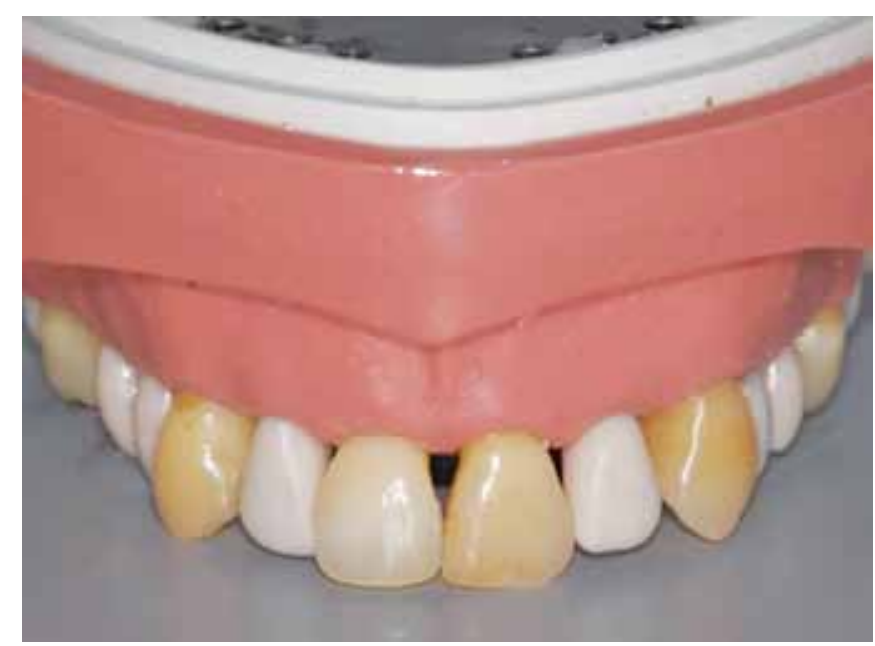

Fig. 1: Set-up of mounted extracted human teeth and artificial typodont teeth with Bonferroni correction applied was used for pair-wise comparison of overall color differences among the three methods. The level of statistical significance was set at 0.05 .

\section{RESULTS}

Repeatability of color measurements of three color parameters $L^{*}, a^{*}$ and $b^{*}$ are summarized in Table 1 . Based on paired-sample t-test, significant differences for several color parameters were noted by measuring method and by operator.

The overall color difference of the three methods of measurements by operator and by tooth material type is summarized in Table 2. For operator A, mean overall color difference- $\Delta \mathrm{E}^{*}$ measured for $\mathrm{CJ}, \mathrm{CT}$ and $\mathrm{FH}$ were $2.86,2.39$ and 2.21 , respectively. There was statistically significant difference in $\Delta \mathrm{E}^{*}$ in $\mathrm{FH}$ vs CJ ( $\left.\mathrm{p}=0.0107\right)$. However, there were no significant differences between FH and CT $(p=0.2829)$ or between CT and CJ $(p=0.1159)$. For operator B mean $\Delta \mathrm{E}^{*}$ for $\mathrm{CJ}, \mathrm{CT}$ and $\mathrm{FH}$ were $2.45,1.95$ and 3.24, respectively. There was a significant difference between FH and CT ( $p=0.0031)$. However, there were no statistically significant differences in $\Delta \mathrm{E}^{*}$ in $\mathrm{FH}$ vs $\mathrm{CJ}$ ( $\mathrm{p}=$ 0.3696) or CT vs CJ ( $\mathrm{p}=0.0809)$. It is noteworthy to point out that there were no significant differences in $\Delta \mathrm{E}^{*}$ for both operators for any measuring method for typodont teeth ( $\mathrm{p}>0.05$ in all instances).

The overall color difference $\left(\Delta \mathrm{E}^{*}\right)$ with three methods of measurement by operator and by human tooth type

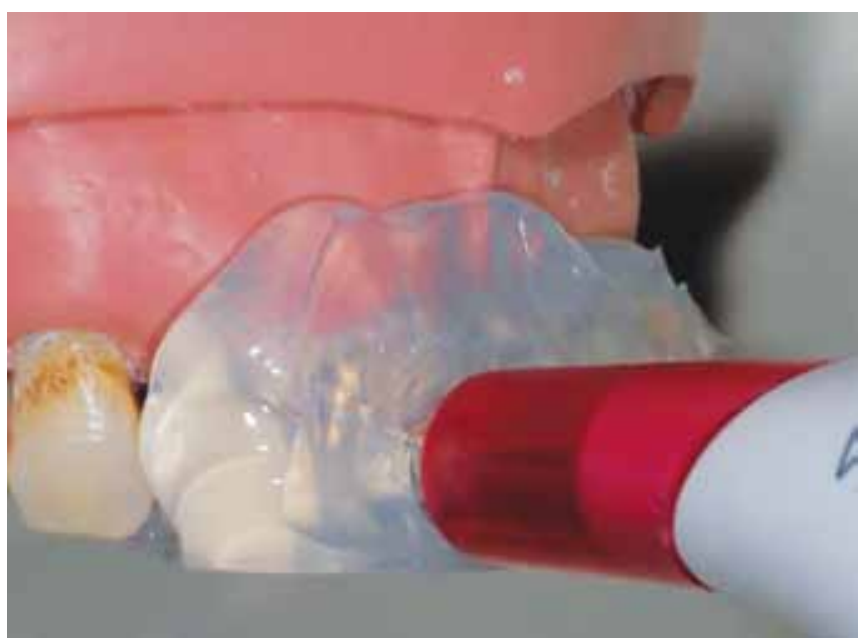

Fig. 2: Tooth color measurement with CJ

Table 1: Evaluation of the difference in the repeatability of color parameters measurements [mean (SD)] obtained from the two operators $(A$ and $B)$ within each measurement method

\begin{tabular}{|c|c|c|c|c|c|c|}
\hline & \multicolumn{2}{|c|}{$L_{2}-L_{1}$} & \multicolumn{2}{|c|}{$a_{2}-a_{1}$} & \multicolumn{2}{|c|}{$b_{2}-b_{1}$} \\
\hline & $\bar{A}$ & $B$ & $\bar{A}$ & $B$ & $A$ & $B$ \\
\hline Custom jig & $1.40(1.63)^{*}$ & $1.17(1.71)^{*}$ & $-0.20(0.45)^{*}$ & $-0.05(0.47)$ & $-0.51(2.62)$ & $0.43(1.96)$ \\
\hline Custom tray & $-0.82(1.73)^{*}$ & $0.12(1.64)$ & $0.13(0.44)$ & $-0.17(0.35)^{*}$ & $-0.34(2.07)$ & $-0.61(1.43)^{*}$ \\
\hline Free hand & $-0.53(2.12)$ & $1.34(3.01)^{*}$ & $-0.14(0.34)^{*}$ & $-0.05(0.92)$ & $-0.51(1.94)^{*}$ & $0.26(3.30)^{*}$ \\
\hline
\end{tabular}

*Statistically significantly different between first and second measurements $(p<0.05)$ using paired-sample t-test 


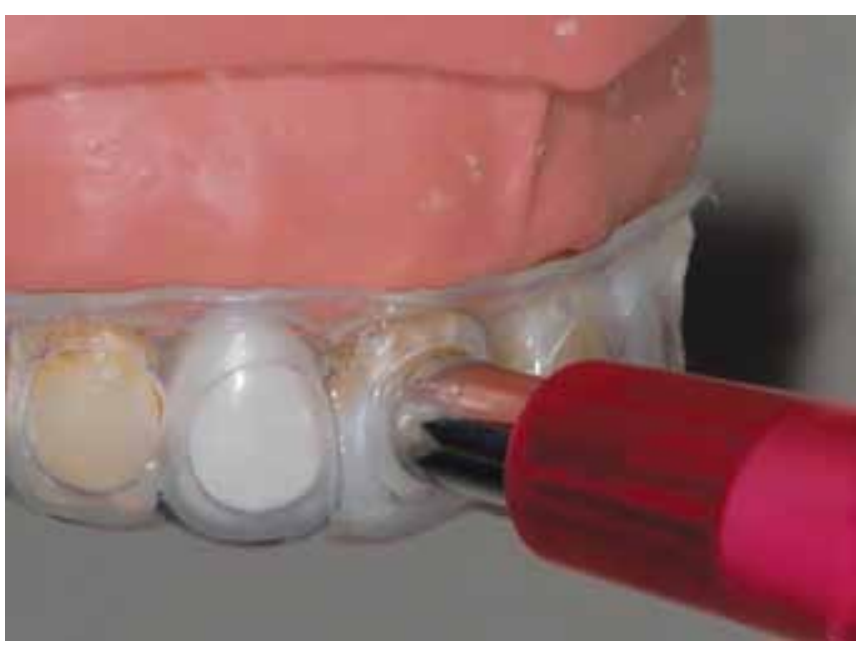

Fig. 3: Tooth color measurement with $\mathrm{CT}$

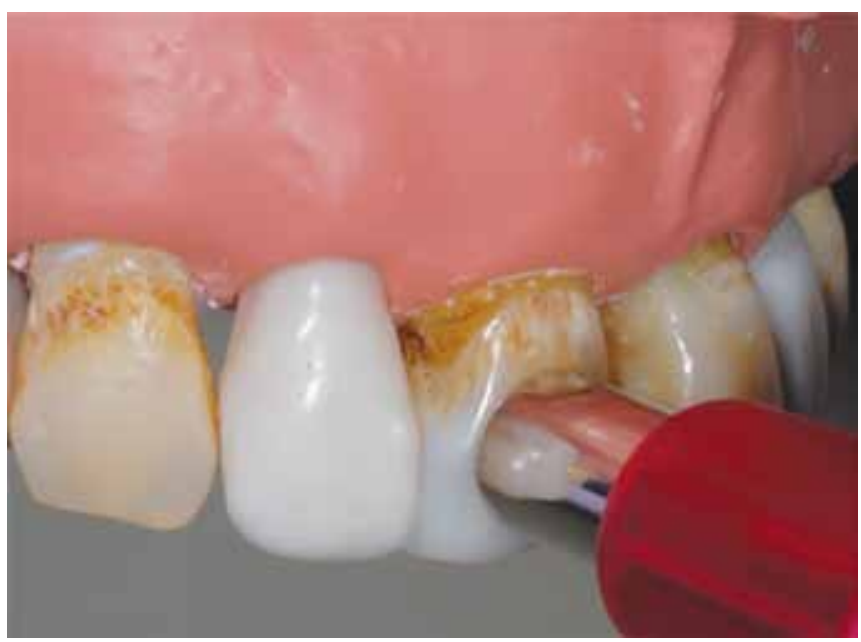

Fig. 4: Tooth color measurement using free hand $\mathrm{FH}$

Table 2: Overall color difference $\left(\Delta \mathrm{E}^{*}\right)$ with three methods of measurement by operator and by tooth material type [mean (SD)]

\begin{tabular}{|c|c|c|c|c|c|c|}
\hline & \multicolumn{2}{|c|}{ Overall $(n=60)$} & \multicolumn{2}{|c|}{ By human teeth $(n=30)$} & \multicolumn{2}{|c|}{ By typodont teeth $(n=30)$} \\
\hline & $\bar{A}$ & $B$ & $A$ & $B$ & $A$ & $B$ \\
\hline Custom jig & $2.86(1.92)^{b}$ & $2.45(1.56)^{a, b}$ & $3.20(2.30)^{b}$ & $2.18(1.33)^{a, b}$ & $2.53(1.42)^{a}$ & $2.72(1.75)^{a}$ \\
\hline Custom tray & $2.39(1.58)^{a, b}$ & $1.95(1.19)^{b}$ & $2.57(1.55)^{a, b}$ & $1.73(1.19)^{b}$ & $2.22(1.61)^{a}$ & $2.17(1.17)^{\mathrm{a}}$ \\
\hline Free hand & $2.21(2.00)^{a}$ & $3.24(3.46)^{a}$ & $2.29(2.39)^{\mathrm{a}}$ & $3.68(4.60)^{\mathrm{a}}$ & $2.13(1.54)^{\mathrm{a}}$ & $2.79(1.69)^{\mathrm{a}}$ \\
\hline
\end{tabular}

*Within column, mean with the same letter was not significantly different $(p>0.05)$ using non-parametric Wilcoxon signed-ranked test with Bonferroni correction

Table 3: Overall color difference $\left(\Delta \mathrm{E}^{*}\right)$ with three methods of measurement by operator and by human tooth type [mean (SD)]

\begin{tabular}{|c|c|c|c|c|c|c|}
\hline & \multicolumn{2}{|c|}{ Central incisors $(n=10)$} & \multicolumn{2}{|c|}{ Canines $(n=10)$} & \multicolumn{2}{|c|}{ Molars $(n=10)$} \\
\hline & $A$ & $B$ & $A$ & $B$ & $A$ & $B$ \\
\hline Custom jig & $2.88(2.14)^{\mathrm{b}}$ & $2.25(1.50)^{a}$ & $2.51(1.49)^{a}$ & $2.37(1.45)^{b}$ & $2.96(1.94)^{a}$ & $2.61(1.67)^{\mathrm{a}}$ \\
\hline Custom tray & $2.48(1.56)^{b}$ & $1.58(0.95)^{a}$ & $1.89(1.28)^{a}$ & $2.37(1.66)^{b}$ & $2.50(1.69)^{a}$ & $2.06(1.13)^{\mathrm{a}}$ \\
\hline Free hand & $1.59(1.19)^{a}$ & $2.11(1.37)^{\mathrm{a}}$ & $2.22(3.09)^{\mathrm{a}}$ & $6.39(6.42)^{a}$ & $2.61(1.95)^{\mathrm{a}}$ & $2.93(2.45)^{\mathrm{a}}$ \\
\hline
\end{tabular}

*Within column, mean with the same letter was not significantly different $(p>0.05)$ using non-parametric Wilcoxon signed-ranked test with Bonferroni correction

is summarized in Table 3. The measurement of canines showed the highest variability with the greatest $\Delta \mathrm{E}^{*}$ of 6.39 units for the free hand held method by operator B, but no significant differences were detected between the three methods in measurements of central incisors and molars. For operator A, no significant difference in measurements of canines and molars was found between the three measurement methods, while the lowest variability with least $\Delta \mathrm{E}^{*}$ of 1.59 units for central incisors was obtained with free hand held method but no significant difference existed between CJ and custom tray held methods.

\section{DISCUSSION}

Recording an accurate baseline shade is extremely difficult due to the complex optical characteristics of tooth color, such as gloss, opacity, transparency, translucency, and optical phenomena, such as metamerism, opalescence and fluorescence. ${ }^{9}$ Since spectrophotometers can detect small differences in color, an important issue of color science in dentistry is to establish a reference value for evaluation of study results in terms of $\Delta \mathrm{E}^{*} .^{1}$ In other words, it is necessary to understand the clinical relevance of $\Delta \mathrm{E}^{*}$ and the ability of the human eye to perceive the color difference. However, this value is subjective and difficult to establish and substantially varies between different groups of dental personnel. ${ }^{10}$ It also varies according to the material type that is compared.

In a study by Kuehni et al that is frequently cited, industrial color matchers evaluated paint and textile specimens and ' $\Delta E^{*}=1$ ' was discernible $50 \%$ of the time. ${ }^{11}$ Seghi et al used translucent porcelain specimens and dental professionals were able to discern a difference $100 \%$ of the time when the $\Delta \mathrm{E}^{*}$ value was greater than $2 .{ }^{12}$ Ragain et al assessed the minimum color difference for discriminating mismatch between composite resin specimens and reported an average acceptability threshold of $2.72 \Delta \mathrm{E}^{*}$ units. $^{13}$ Johnston and Kao in their clinical study showed that the average color difference between compared teeth and composite restorations rated as a match in the oral environment was 3.7 units. ${ }^{14}$ Thus with different threshold values reported in different studies it 
was stressed that one single value was probably not the key solution to the problem, and that a differentiation between perceptibility (the difference that can be identified by the human eye) and acceptability (the difference that is considered tolerable) was proposed. ${ }^{1}$ Douglass et al determined the valid acceptability and perceptibility tolerances for shade mismatch in a clinical study using spectroradiometric instrumentation and reported that the predicted color difference at which $50 \%$ of the dentist observers could perceive a color difference was $2.6 \Delta \mathrm{E}^{*}$ units. ${ }^{15}$ Whereas the predicted color difference at which $50 \%$ of the subjects would remake the restoration due to color mismatch was $5.5 \Delta \mathrm{E}^{*}$ units. In order to provide a range for the identification of the perceivable $\Delta \mathrm{E}^{*}$ value, Vichi et al proposed three different intervals for distinguishing color differences. $\Delta \mathrm{E}^{*}$ values of less than 1 unit were regarded as not appreciable by the human eye; $\Delta \mathrm{E}^{*}$ values greater than 1 and less than 3.3 units were considered appreciable by skilled operators, but clinically acceptable; $\Delta \mathrm{E}^{*}$ values greater than 3.3 were considered perceivable by untrained observers. ${ }^{16}$

Despite much effort on the identification of a $\Delta \mathrm{E}^{*}$ value in terms of perceptibility and acceptability the values are still controversial and require further investigation. The aim of this study was to evaluate the repeatability of an intraoral spectrophotometer using the conventional free hand held method and with the use of two different types of positioning devices. Based on the results:

The first hypothesis was rejected: the repeatability of color measurement for the three color parameters was statistically different by measurement method and by operator.

The second hypothesis was also rejected: the overall color difference observed, when comparing the first and second color measurements were statistically different by measurement method and by operator. The overall color change observed using different methods was different between the two operators. For operator A the free hand and custom tray worked well, whereas for operator B the custom tray and CJ increased the repeatability of the color measurements. The overall range of 1.95 to $3.24 \Delta \mathrm{E}^{*}$ units is comparable to other studies that used a negative control and found a range from 0.86 to 2.25 and 2.2 to $3.2 \Delta \mathrm{E}^{*}$ units. ${ }^{17,18}$ This is important in determining the critical threshold for tooth whitening studies-suggesting that even without any treatment an overall color change of up to $3.2 \Delta \mathrm{E}^{*}$ units can result due to variability in color measurements. Our study results are not in accordance with another study that reported a short term repeatability of less than $0.7 \Delta \mathrm{E}^{*}$ units when the CJ was used. ${ }^{8}$ A possible reason for this disparity may be attributed to a difference in operator training for the two studies. The operator of our study consisted of sophomore students that were not experienced in color measurements and were trained just prior to the study. Another possible explanation may also be the difference in design of the jig. In our study, the CJ was fabricated such that four teeth could be measured with one jig, whereas the other study used a CJ for a single tooth.

The third hypothesis was rejected: there was significant difference of overall color difference by type of tooth material with typodont teeth showing less variation for both operator and measuring method. This is in accordance with other studies that have emphasized the difficulty of obtaining consistent color measurement results in human teeth not only due to positioning errors but also due to potential dehydration issues, which may not be an issue with typodont teeth. ${ }^{19,20}$ It is also important to mention that within human teeth the highest variability was observed in canines which inherently manifest a more convex buccal surface when compared to the flat surface of central incisors and molars. This is also a commonly noted disadvantage of intraoral spectrophotometers as their high repeatability is mainly limited to flat surfaces.

\section{CONCLUSION}

Within the limitations of this study, it can be concluded that repeatability of color measurements were different among the three measuring methods by operator. Overall, the custom tray method worked well for both operators and typodont teeth showed less variation in color measurement than extracted human teeth.

\section{ACKNOWLEDGMENT}

This study was supported by the University of Iowa Dental Research Grant.

\section{REFERENCES}

1. Vichi A, Louca C, Corciolani G, Ferrari M. Color related to ceramic and zirconia restorations: a review. Dent Mater 2011; 27:97-108.

2. Paul S, Peter A, Pietrobon N, Hämmerle CH. Visual and spectrophotometric shade analysis of human teeth. J Dent Res 2002;81:578-582.

3. Dozic A, Kleverlaan CJ, El-Zohairy A, Feilzer AJ, Khashayar G. Performance of five commercially available tooth color measuring devices. J Prosth 2007;16:93-100.

4. Sarafianou A, Kamposiora P, Papavasiliou G, Goula H. Matching repeatability and interdevice agreement of 2 intraoral spectrophotometers. J Prosthet Dent 2012;107:178-185.

5. Chu SJ. Use of a reflectance spectrophotometer in evaluating shade change resulting from tooth-whitening products. J Esthet Restor Dent 2003;15(Suppl 1)S42-48.

6. Kim-Pusateri S, Brewer JD, Dunford RG, Wee AG. In vitro model to evaluate reliability and accuracy of a dental shadematching instrument. J Prosthet Dent 2007;98:353-358.

7. Karamouzos A, Papadopoulos MA, Kolokithas G, Athanasiou AE. Precision of in vivo spectrophotometric color evaluation of natural teeth. J Oral Rehab 2007;34:613-621. 
8. Ontiveros JC, Paravina R. Color change of vital teeth exposed to bleaching performed with and without supplementary light. J Dent 2009;37:840-847.

9. Hunter RS. The measurement of appearance. 2nd ed. New York: John Wiley and Sons Inc.; 1987.

10. Sim CP, Yap AU, Teo J. Color perception among different dental personnel. Oper Dent 2001;26:435-439.

11. Kuehni FG, Marcus RT. An experiment in visual scaling of small color differences. Color Res App 1979;68:1755-1759.

12. Seghi RR, Johnston WM, O'Brien WJ. Performance assessment of colorimetric devices on dental porcelains. J Dent Res 1989;68:1755-1759.

13. Ragain JC Jr, Johnston WM. Minimum color differences for discriminating mismatch between composite and tooth color. J Esthet Restor Dent 2001;13:41-48.

14. Johnston WM, Kao EC. Assessment of appearance match by visual observation and clinical colorimetry. J Dent Res $1989 ; 68: 819-822$.
15. Douglas RD, Brewer JD. Acceptability of shade differences in metal ceramic crowns. J Prosthet Dent 1998;79:254-260.

16. Vichi A, Ferrari M, Davidson CL. Color and opacity variations in three different resin-based composite products after water aging. Dent Mater 2004;20:530-534.

17. Kwon S, Li Y, Oyoyo U, Aprecio RM. Dynamic model of hydrogen peroxide diffusion kinetics into the pulp cavity. J Contemp Dent Pract 2012;13:440-445.

18. Kwon S, Meharry M, Oyoyo U, Li Y. Efficacy of Do-ItYourself Whitening as Compared to Conventional Tooth Whitening Modalities: An In Vitro Study. Operative Dentistry 2014;40:E21-27.

19. Geisinger S, Kwon S, Qian F. Employment of reservoirs in at-home whitening trays: efficacy and efficiency in tooth whitening. J Contemp Dent Pract 2015;16:383-388.

20. Russell MD, Gulfraz M, Moss BW. In vivo measurement of colour changes in natural teeth. J Oral Rehabil 2000;27: 786-792. 